\title{
ReMed: les crises ont de nombreux visages
}

\section{Iris Leu, Peter Christen}

Pour le Comité de direction de ReMed

Les médecins aussi connaissent des situations de crise. Celles-ci surviennent de façon inattendue et mettent le quotidien sens dessus dessous. Il peut s'agir d'un conflit au travail qui dégénère subitement, d'une erreur de traitement qui ébranle la confiance en soi ou de difficultés relationnelles qui peuvent engendrer des sentiments dépressifs.

Dans de telles situations, ReMed apporte rapidement son soutien sans bureaucratie inutile. Lorsque vous prenez contact avec le réseau de soutien, une équipe de conseillers expérimentés vous répondra dans les 72 heures pour discuter de votre situation personnelle. Le burnout n'épargne pas les médecins, bien au contraire, car ceux-ci sont particulièrement vulnérables. On estime qu'environ $20 \%$ d'entre eux souffrent de ce syndrome et que $78 \%$ sont insatisfaits sur le plan professionnel. Selon le Dr Thomas Bergner, spécialiste en pratique privée et auteur d'un livre sur l'épuisement professionnel chez les médecins, ce phénomène marginal au départ s'est répandu comme une traînée de poudre dans le domaine de la santé.

Or, la diversité des facteurs qui mènent à la maladie et le nombre important de symptômes associés rendent le diagnostic précoce difficile. ReMed est

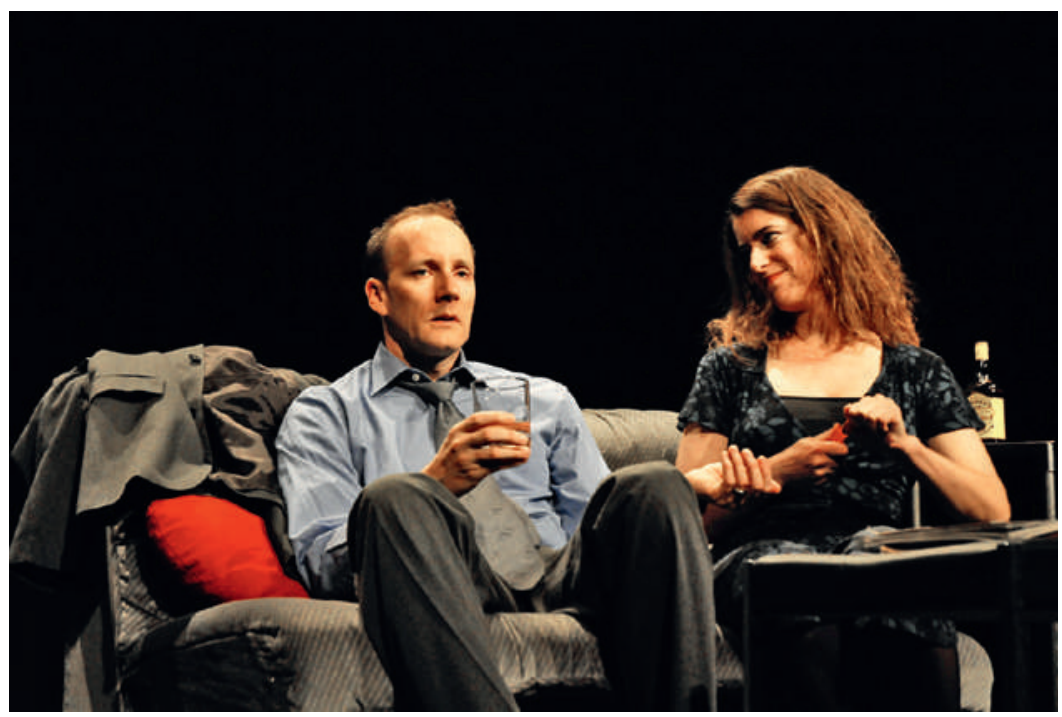

Le recours à I'alcool: un signe d'épuisement, de stress et de burnout. conscient de ce problème et ne se contente pas seulement de proposer des consultations mais axe aussi sa démarche sur la prévention.

Les ateliers et formations continues au moyen de pièces de théâtre interactives sont un bon outil de prévention et de sensibilisation. Grâce à l'intervention ciblée d'un animateur, ces pièces de théâtre interactives permettent aux spectateurs d'influer selon leur propre ressenti sur la manière d'agir des différents personnages. Le public intervient activement sur ce qui se passe sur scène et teste en direct sa vision des choses. Il voit ainsi immédiatement les conséquences de ses propres actions.

Les médecins peuvent se mettre dans la peau d'un personnage qu'ils connaissent bien et chercher des solutions.

Cette offre, qui permet d'exercer sa façon d'agir dans des situations conflictuelles, s'adresse aux sociétés de discipline, aux sociétés cantonales de médecine, aux organisations de médecins, aux institutions sociales, aux établissements de formation et aux entreprises de même qu'à un public plus large.

Différentes représentations théâtrales interactives ont eu lieu en Suisse romande et alémanique ces dernières semaines et ces derniers mois en collaboration avec ReMed. Nous en présentons deux ci-après.

\section{«Knotenpunkt» à l'Hôpital cantonal de Glaris: une session de formation continue pour les médecins de famille}

$\mathrm{Au}$ travers de la pièce intitulée «Der Nächste bitte» («Au suivant!»), quatre comédiens ont rejoué une scène tirée de la vie quotidienne d'un médecin, en montrant avec pertinence comment la situation se dégrade peu à peu à l'insu du protagoniste et de ses proches:

- Le médecin passe tout son temps au cabinet;

- il néglige sa famille;

- ses amis lui tournent le dos. 
Le burnout est l'un des visages de la crise. Ce médecin, qui n'arrive plus à faire preuve d'empathie envers ses patients, ne parle plus à sa femme et n'a plus l'énergie de lire une histoire à sa fille, néglige ses amis et se tourne de plus en plus souvent vers la bouteille.

Cette pièce vise à montrer au public quand et comment les proches peuvent et doivent réagir et quand il leur faut dire stop pour protéger la personne concernée. Lorsque Fra Zeller, l'animatrice, dit «Stop», les acteurs s'immobilisent et le spectateur est invité à jouer lui-même la solution qu'il propose. Ce type de

\section{La troupe a su, avec professionnalisme et humour, inciter les médecins à réfléchir à leur propre syndrome d'épuisement.}

théâtre permet aux médecins dans le public de se mettre dans la peau d'un personnage qu'ils connaissent bien et de chercher des solutions en collaboration avec les autres spectateurs dans le but de réduire les risques de la crise et de ressentir les conséquences de ses propres actes.

Cette représentation a rencontré un franc succès à Glaris et les spectateurs étaient enthousiastes et motivés à intervenir selon leur vision des choses, même s'ils ont ensuite réalisé combien il était difficile d'agir au mieux en situation de crise. L'apéritif qui a clos la manifestation a permis aux participants d'échanger leur expérience et de nouer de nouveaux contacts.

Quelques informations sur la troupe théâtrale «Knotenpunkt» Les crises ont de nombreux visages. En plus du burnout, la troupe traite également d'autres sujets tabous tels que l'abus d'alcool ou la dépression, les démences et la maladie d'Alzheimer, la violence envers les personnes âgées, le cancer, les lésions cérébrales ou encore les angoisses et autres phobies. La troupe propose également des coachings sur mesure sous forme de petites pièces pour des problèmes aigus.

Dans ces scènes très réalistes, le public peut expérimenter et interagir avec les acteurs et le public. Cette offre permet de s'entraîner à la manière d'agir dans des situations conflictuelles. Elle s'adresse aux organisations médicales, aux institutions sociales, aux établissements de formation, aux entreprises ainsi qu'à un public plus large. Vous trouverez de plus amples informations sur le site www.theater-knotenpunkt.ch.
Quelques informations sur la Compagnie "Le Caméléon» La compagnie "Le Caméléon" est active sur les scènes romandes depuis une vingtaine d'années et, depuis 2012, également en Suisse alémanique. Elle se compose de 17 comédiens professionnels romands et de sept comédiens alémaniques, dont la plupart ont suivi une deuxième formation dans le domaine social.

La troupe s'inspire de la méthode développée par Augusto Boal dans son théâtre des opprimés qui permet de traiter des sujets difficiles de manière interactive et avec humour, sans être moralisateur. Au moyen d'animations interactives, la compagnie vise à mobiliser les ressources propres à chaque individu.

Hormis ses représentations de "théâtre-forum", la compagnie intervient également lors de séminaires, de congrès et de ses sions de formation destinées à différents groupes professionnels. Vous trouverez un aperçu de leurs offres sur le site www. lecameleon.ch.

\section{Théâtre-forum "Le Caméléon" au congrès Quadrimed de Crans-Montana}

Dans la pièce "Comment allez-vous docteur?», le quotidien des médecins est passé au crible des comédiens de la troupe Le Caméléon. Le public a l'occasion de découvrir le quotidien du Dr Herbert en 2048 (!). Assistant à ses débuts, il ouvre ensuite son propre cabinet et est vite dépassé par les événements: urgences, patients difficiles, maladie non diagnostiquée, charge administrative trop importante. C'en est trop. Il se soigne au whisky et aux cocktails médicamenteux, néglige lui aussi ses proches et arrive au bout de ses forces.

Dans cette pièce, les médecins du public sont interpelés avec humour par le "Joker» Carlos Henriquez qui les invite à se mettre dans la peau du Dr Herbert, de ses collègues et de sa femme pour essayer de trouver d'autres attitudes ou voies pour aboutir au bon équilibre entre vie privée et professionnelle. La troupe a su, avec professionnalisme et humour, inciter les médecins présents à réfléchir à leur propre syndrome d'épuisement et à échanger leurs expériences.

\section{N'hésitez pas à contacter ReMed}

Si vous êtes intéressés à mettre sur pied une représentation théâtrale interactive dans votre organisation ou institution, ou si vous souhaitez y assister ou obtenir de plus amples informations, veuillez vous adresser à info[at]swissremed.ch. 